\title{
A New Systematic Encoding Circuit of Hamming $(15,11)$ Using Low-Power XOR-FS-GDI Based Logic Gate: Fast Computing and Efficient Area FEC
}

Mohsen El-Bendary ( $\sim$ engmohsen2004@yahoo.com )

Helwan University https://orcid.org/0000-0002-2425-4967

O El-Badry

Sohag University

\section{Research Article}

Keywords: Low power Hamming codes, Data encoding, 65 nm Technology, FS-GDI, Hardware simplicity

Posted Date: December 22nd, 2021

DOI: https://doi.org/10.21203/rs.3.rs-1144966/v1

License: (c) (i) This work is licensed under a Creative Commons Attribution 4.0 International License.

Read Full License 


\title{
A New Systematic Encoding Circuit of Hamming $(15,11)$ Using Low-Power XOR-FS-GDI Based Logic Gate: Fast Computing and Efficient Area FEC
}

\author{
Mohsen A. M. El-Bendary ${ }^{1}$ O. El-Badry ${ }^{2}$ \\ ${ }^{1}$ Faculty of Technology and Education, Helwan University, Egypt. \\ ${ }^{2}$ Department of Electrical Technology, Sohag University, Sohag, Egypt
}

\begin{abstract}
:-
Due to the power efficiency importance of digital signal processing and data protection in different communications systems, this paper proposes an efficient design of different Hamming Codes utilizing Full Swing- Gate Diffusion Input (FS-GDI) approach. The proposed codes design aims to improve the power efficiency and the required area through reducing the required number of transistors. FS-GDI is a new low power VLSI design approach, it is a power effective approach for realizing the different logic gates. In this work, the Hamming codes $(11,7)$ and $(15,11)$ are designed by utilizing the original GDI, FS-GDI and the traditional CMOS approaches. The amount of consumed power, delay time, Power Delay Product (PDP) and hardware simplicity-Number of Transistors (No. Ts) are employed as a metrics for evaluating the efficiency of the proposed design compared to the traditional design. The design simulation experiments are executed utilizing Cadence Virtuoso simulator package under $65 \mathrm{~nm}$ technology. The simulation experiments revealed these proposed codes achieve delay time reduction by $52.91 \%$ and $10 \%$ for Hamming codes $(7,4)$ and $(11,7)$, respectively On the other hand, the Hardware $(\mathrm{H} / \mathrm{W})$ of these codes became more simple where the H/W simplicity of the used Hamming codes is reduced $50 \%$ CMOS approaches respectively. From the results analysis, the proposed design achieves efficient power and the delay optimizing of Hamming codes utilizing the FS-GDI approach. On the other hand, the power consumption and area in communications systems due to the encoding process can be reduces .
\end{abstract}

Keywords:- Low power Hamming codes, Data encoding, $65 \mathrm{~nm}$ Technology, FS-GDI, Hardware simplicity.

\section{Introduction}

The channel coding techniques play important role in the transmitted data protection over the wireless communications channels. The amount of consumed power limits the utilizing of error coding techniques and the powerful data security techniques due to its 
computational complexity [1]. In this paper, low power error control hamming codes design is proposed using FS-GDI approach. The FS-GDI approach is power efficient low power VLSI logic style compared to the traditional approaches [2-10].

In [11] the complexity in the wireless communications due to the amount of the transmitted data encoding is analyzed based on the used error control code is the Convolutional codes and the Hamming code. The presented analysis in this paper proved that the complexity is related to the amount of required/consumed power. That means with increasing the computational complexity, the consumed power increases. On the other hand. the power efficiency of the communications systems is considered in several research papers [12-19]. The power is main constraint for the application of the data protection techniques. Hence, design low power error control schemes using the power efficient approach will be increasing the capability of data protection techniques using and enhancing the power efficiency of the systems in general [20].

In this paper, different Hamming codes design is proposed based on GDI and FSGDI approaches. The proposed codes design is compared with the traditional CMOS approach utilizing different metrics such as the delay time, consumed power, Power Delay Product (PDP) and number of transistors which are required for implementing the Hamming codes by the different traditional CMOS, GDI and FS_GDI approaches. The simulation experiments prove the superiority of the proposed Hamming code design using FS-GDI compared to the traditional approach. The software package which is used for performing the design and the simulation experiments is Cadence Virtuoso simulator [21-24].

In the following, the rest of paper is presented as follows: section 2 presents the low power VLSI approaches and its sub-approaches. GDI approach Development and Versions are introduced in section 3. In section 4, the complexity due to the encoding process is discussed. The proposed work description of different Hamming implementation and experiments codes are presented in section 5. In section 6, result analysis is presented. the conclusion is introduced in section 7.

\section{Low Power VLSI Approaches Overview}

The different styles of the VLSI are presented in this section. In the following, the different approaches advantages and disadvantages are discussed. Figures 1 and 2 give AND and XOR logic gates realizing using the different approaches, respectively. 


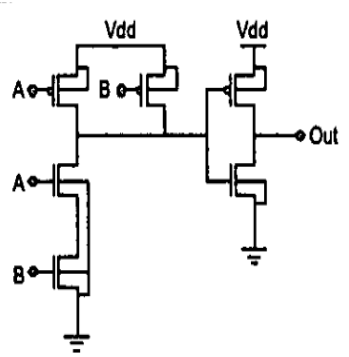

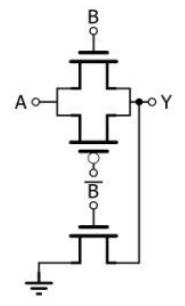

b. AND Gate PTL-based

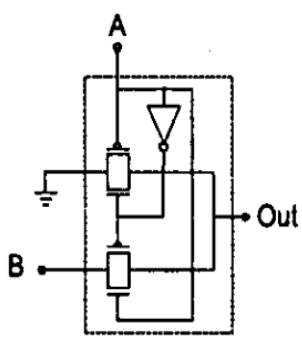

c. AND Gate TG-based

a. AND Gate CMOS-based

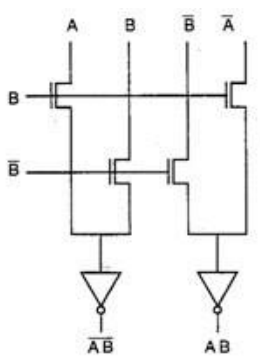

(a)

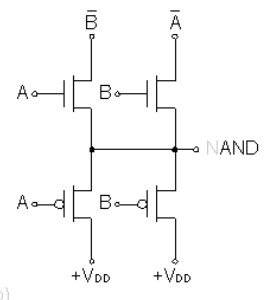

e. AND Gate DPL-based

d. NAND/AND Gate CPL-based

Fig.1 Different traditional approaches for realizing AND logic gate.

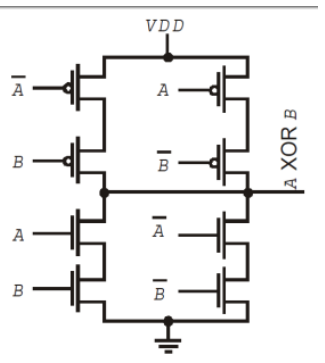

a. XOR Gate CMOS Logic

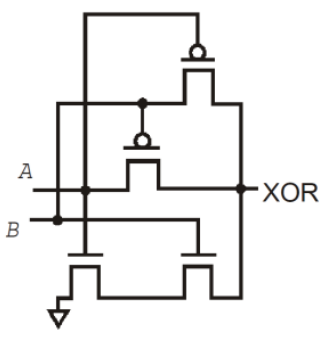

b. XOR Gate -PTL Logic

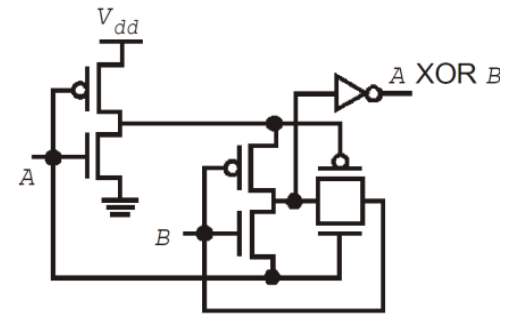

c. XOR Gate -TG Logic

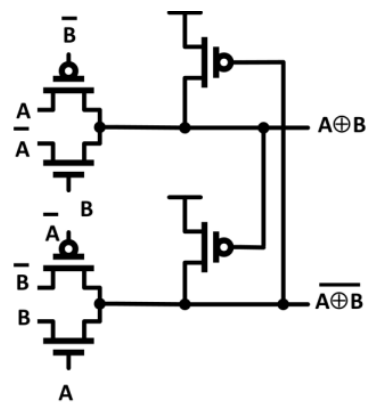

d. XOR/XNOR Gate- CPL Logic

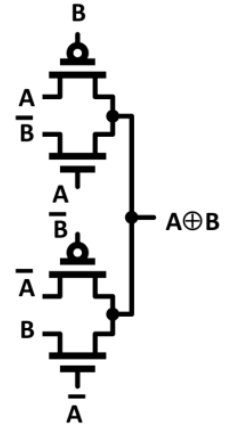

e. XOR Gate-DPL Logic

Fig 2. XOR Gate Realizing using Different approaches 
The Complementary symmetry metal-oxide-semiconductor CMOS is the most popular approach, it has several advantages such as, low noise margin, high speed, low power, easy to develop, common in most chips design of VLSI. Also, it suffers some problems such as, high power dissipation, large number of transistors/area, long delay time, power consumption high. The second common approach is the Pass Transistor Logic (PTL), it is popular as CMOS approach, its features as decreased silicon area, speed, reduced power consumption, slower at reduced power, significant power dissipation [25-28].

The third approach is the Transmission Gate (TG), its feature is less transistor for implementing complex gates, PMOS and NMOS combination avoid noise margin, power dissipation, switching, require control, limited TG cascade. The fourth approach is the Complementary Pass-Transistor logic- (CPL), ( N-MOSFET approach), its advantages is high speed, low i/p capacitance and easy to implement complex logic by NMOD net. It is drawbacks is drop in threshold voltage, static power consumption and delay increases with long pass-transistor chains. The Double Pass-transistor Logic (DPL) approach is the modified of CPL approach, it has some advantages as well-balanced input capacitance No drop voltage, no need buffers, full swing and low power, its main problems are large area and inverters need [29-32].

\section{GDI approach Development and Versions}

A long fourteen years, Morgenshtein et al. presented the GDI approach for low power VLSI implementing. In 2002, 2010 and 2014, the original cell of GDI, modified GDI and Full Swing GDI have been presented, respectively by Morgenshtein. Gate diffusion input (GDI) is a new low power technique using small silicon chip area for digital VLSI design compared to another logic style proposed by Morgenshtein. This approach aimed to improve the power consumption and area as well as propagation time of the logic gates implementing compared to the traditional approaches [33-35].

In 2001, the GDI approach was invented, it allowed complex gate implementing by two transistors. This method is suitable for the design of fast, low-power circuits, using a reduced number of transistors (as compared to CMOS and existing PTL techniques) as shown in Table 1 [33]. As shown in this table the different logic gates hardware can be simplified by reducing the number of transistors to be more power efficient. 
Table 1 Transistors count between GDI and CMOS for various functions.

\begin{tabular}{|c|c|c|}
\hline $\begin{array}{c}\text { Different Realized } \\
\text { Function }\end{array}$ & $\begin{array}{c}\text { CMOS } \\
\text { Approach }\end{array}$ & $\begin{array}{c}\text { GDI } \\
\text { Approach }\end{array}$ \\
\hline $\mathrm{F} 1=\bar{A} \mathrm{~B}$ & 6 & 2 \\
\hline $\mathrm{F} 2=\bar{A}+\mathrm{B}$ & 6 & 2 \\
\hline OR & 6 & 2 \\
\hline AND & 6 & 2 \\
\hline NAND & 4 & 4 \\
\hline NOR & 4 & 4 \\
\hline MUX & 12 & 2 \\
\hline NOT & 2 & 2 \\
\hline
\end{tabular}

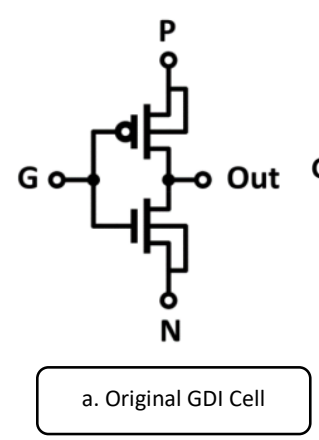

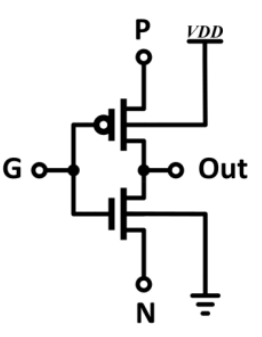

b. Modified GDI Cell

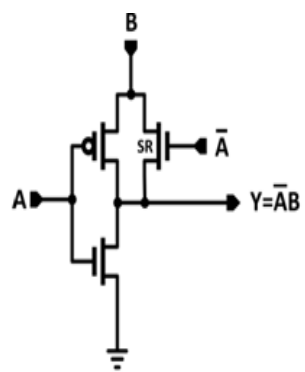

c. NAND Gate FS-GDI

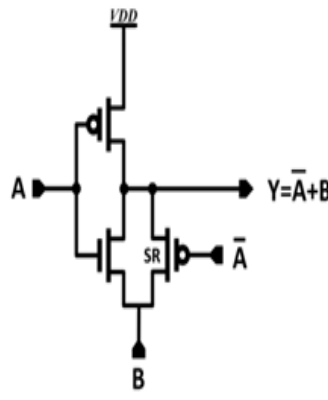

d. NOR Gate FS-GDI

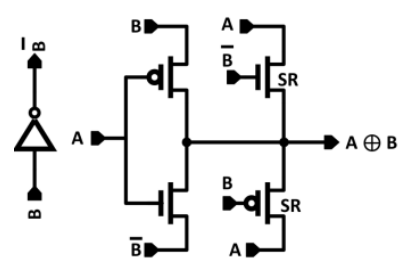

e. XOR Gate FS-GDI

Fig. 3 Cell of GDI approach developing, a-Original GDI cell, 2001, b-

Modified GDI cell 2010, and d-FS-GDI, 2014.

Figure 3 shows the GDI approach versions from 2001 to 2014. Fig. 2a gives the original cell of GDI, Fig. $2 b$ presents the modified cell, while the last version of GDI approach is shown in Fig. 2c. Using GDI technique allowed improvement in power 
consumption, propagation delay and area of VLSI digital circuits compared to CMOS and pass transistor logic (PTL) techniques. PTL and GDI both suffered from reduced voltage swing at their outputs due to threshold drops. Problems of the first GDI cell are solved by Morgenshtein in 2014 through proposing the Full Swing GDI (FS-GDI) based on a single swing Restoration Transistor (SR) as an alternative to another method. The SR transistor ensures full swing operation [35].

The logic gates such as AND, OR and XOR gates can be implemented with full swing operation can be achieved but with increasing the transistor count from 2 to 3 compared to the original GDI design. In [36], Shoba and et al proposed a new design of AND, OR and XOR logic gates based on full swing GDI technique, which are used to implement three designs of full adder [36]. However, the three full adders are successfully realized using full swing gates with a significant improvement in their performance [37-38].

\section{Encoding Process Complexity}

As mentioned in section 1, the computational complexity is the amount of operations in the systems for perfuming its function. The complexity is directly related to the amount of the required power and the process time, it is called also time complexity. The error control codes utilizing for data protection is essential issue. The computational complexity of these codes differs according to its type and capabilities. The block codes such as Hamming codes complexity is determined based on its data-word (k) and codeword (n) length, in general it simpler than the Convolutional codes. The Convolutional codes encoding/decoding have high complex compared to the block codes [40]. In this paper, the simpler block codes (Hamming codes) are chosen for evaluating the proposed design of data protection techniques by the FSGDI approach.

The complexity basis of error control codes is presented in this section. Block codes complexity is limited and lower than Convolutional codes [20]. Mostly, complexity of block codes is controlled by two factors, which are the input length of the encoder $(\mathrm{k})$ and the codeword the output of the encoder (n). In the block codes the length of the processed data is not important factor, the packet already segmented to small parts each one of them has length of data word. In the Convolutional codes, the complexity is controlled by many factors, length of input it is the processed data in the same time interval, the length of output, the length of the processed data "packet length" and finally, length of the memory in Convolutional encoder. In fact, the computational complexity of the Convolutional codes increases with the constraint length increasing [41-42]. 
In this table, the extra computational complexity related to the proposed technique is considered and the complexity related to the memory length of the convolutional encoder also. As shown in the numerical analysis of the hardware complexity of the traditional CMOS approach and FS-GDI approach Hamming codes in Table 4, it proves that the superiority of the proposed technique complexity especially with the longer data-word packets processing.

\section{The Proposed Scheme Description}

In this section, the different Hamming codes $(7,4),(11,7)$ and $(15,11)$ implementation approaches are presented. The FS-GDI approach are proposed for implementing the Hamming codes as an approach for achieving power efficient data encoding tool. In Table 2, the general characteristics of the Hamming codes are tabulated.

Table 2 Different Hamming codes characteristics with respect to $\mathrm{k}, \mathrm{n}$ and $\mathrm{R}$

\begin{tabular}{|l|c|c|c|c|}
\hline $\begin{array}{c}\text { Coding Scheme } \\
\text { Name }\end{array}$ & $\begin{array}{c}\text { Parity bits } \\
\text { (Extra Bits) }\end{array}$ & Data bits (k) & Codedword (n) & Code Rate=k/n \\
\hline Hamming Code (7,4) & 3 & 4 & 7 & $4 / 7=0.571$ \\
\hline Hamming Code(11,7) & 4 & 7 & 11 & $11 / 7=0.636$ \\
\hline Hamming Code (15,11) & 4 & 11 & 15 & $11 / 15=0.733$ \\
\hline Hamming Code (n, k) & $m=n-k$ & $k=2^{m}-m-1$ & $n=2^{m}-1$ & $R=2^{m}-m-1 / 2^{m}-1$ \\
\hline
\end{tabular}

\subsection{The Proposed FS-GDI Hamming Code $(7,4)$ Design Implementing}

In this section, simple data protection Hamming code $(7,4)$ error control scheme is implemented and realized by the FS-GDI approach for enhancing its power efficiency and hardware simplicity. In this error control code, 7 number represents the number of output of Hamming encoder bits it is 7 bits. The number 4 refers to the number of bits encoder input, it is 4 bits in this encoder.

Hamming Algorithm mechanism:- The Hamming code algorithm is working as described following steps:

- The parity bits (P) are generated and added to (k) bits data-word to form the codeword of $(n) n=k+P$ bits.

- The $\mathrm{P}$ bits are generated by the simple equations from the input data (data-word).

The data-word is the input of the encoder circuit, it performs XOR operations on the $\mathrm{k}$ data-word, hence the required (p) parity bits generated. Parity bits and data bits together form the code word. An encoder circuit of Hamming code for 4-bit data word is shown Fig. 4. 
Following this circuit pattern, we can design an encoder circuit of hamming code for 7-bit data word and it is implemented in cadence virtuoso tool.

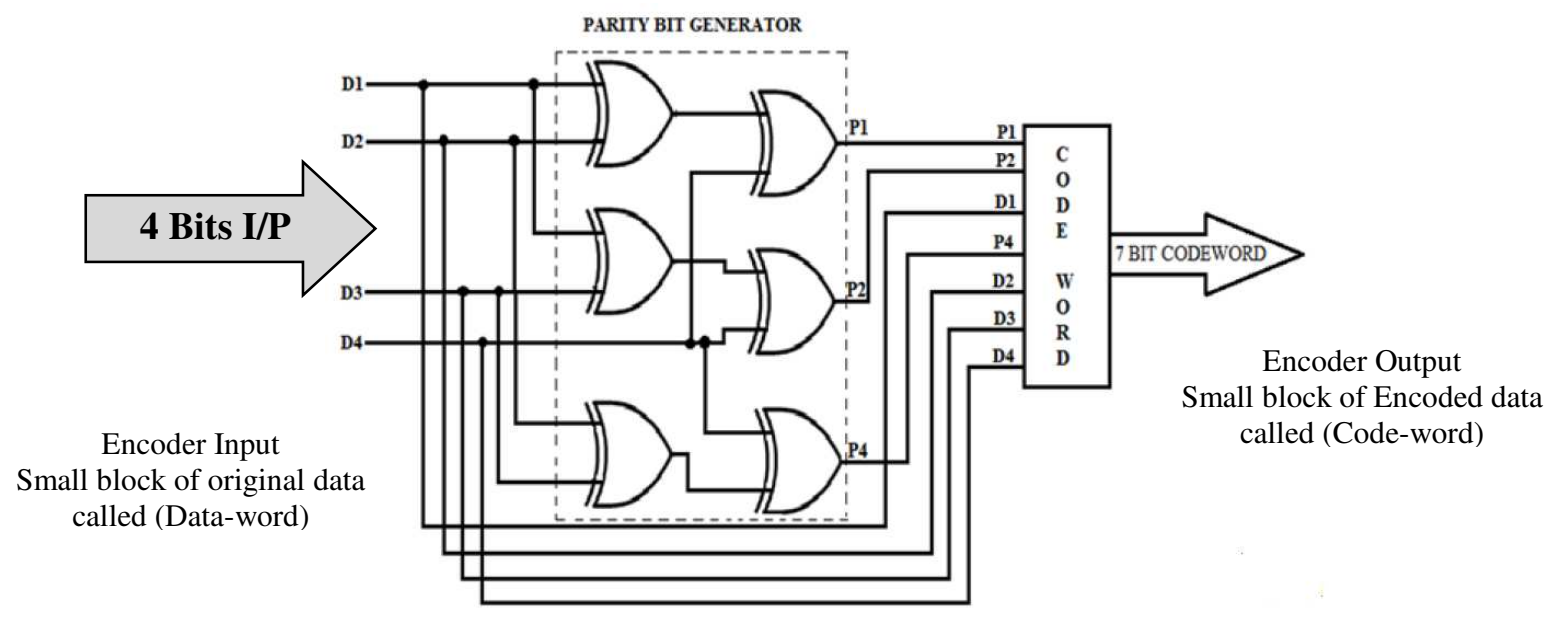

Fig. 4 Hamming Encoder Circuit(7, 4) Contents

Fig. 4 consists of 4-bit data word, parity bit generator and 7-bit code word. The 4-bit data word is applied as an input to the encoder circuit, now the encoder output consist of 7bits i.e. 4-data bits $\mathrm{D}_{1}, \mathrm{D}_{2}, \mathrm{D}_{3} \& \mathrm{D}_{4}$ and 3-parity bits $\mathrm{P}_{1}, \mathrm{P}_{2} \& \mathrm{P}_{4}$, as in Eqs (1-3):

$$
\begin{aligned}
& \mathrm{P}_{1}=\mathrm{D}_{1} \oplus \mathrm{D}_{2} \oplus \mathrm{D}_{4} \\
& \mathrm{P}_{2}=\mathrm{D}_{1} \oplus \mathrm{D}_{3} \oplus \mathrm{D}_{4} \\
& \mathrm{P}_{4}=\mathrm{D}_{2} \oplus \mathrm{D}_{3} \oplus \mathrm{D}_{4}
\end{aligned}
$$

The Codeword : $\mathrm{D}_{4} \mathrm{D}_{3} \mathrm{D}_{2} \mathrm{P}_{4} \mathrm{D}_{1} \mathrm{P}_{2} \mathrm{P}_{1}$

The data word is applied as an input to the encoder circuit which performs XOR operations on the given data word thus the required parity bits are generated from the parity bit generator. Parity bits and data bits together form the code word. An encoder circuit of hamming code for 4-bit data word is shown in Fig. 4. The Hamming encoder consists of 4-bit data word, parity bit generator and 7-bit code word. The 4-bit data word is applied as an input to the encoder circuit, now the encoder output consists of 7-bits i.e. 4-data bits $\mathrm{D}_{1}, \mathrm{D}_{2}, \mathrm{D}_{3} \&$ $\mathrm{D}_{4}$ and 3-parity bits $\mathrm{P}_{1}, \mathrm{P}_{2} \& \mathrm{P}_{4}$, as in Eqs (1-3).

\section{a. Testing the Proposed design of Hamming Encoder (7, 4) Performance}

Simulations were done using the spectre based Cadence Virtuoso simulator with a power supply of $1 \mathrm{~V}$ and frequency $100 \mathrm{M} \mathrm{HZ}$, the size of PMOS is twice the NMOS 
transistor size $\mathrm{Wp} / \mathrm{L}=240 / 60, \mathrm{Wn} / \mathrm{L}=120 / 60$ (PMOS and NMOS) respectively to achieve the best power and delay performance. Each component is analyzed in terms of propagation delay, power dissipation, and their product. The propagation delay is measured by accounting the time taken from $50 \%$ of the input voltage swing to $50 \%$ of the output voltage swing for each transition. Table 2 shows the values of the evaluating metrics of the proposed implementing approach of Hamming code $(7,4)$.

Table 2 Metrics values of the proposed Hamming Encoder $(7,4)$ design with respect to the different approaches: Proposed \& Traditional

\begin{tabular}{l|cccc}
\multirow{2}{*}{ Utilized Approach } & \multicolumn{4}{|c}{ Metrics of Performance Evaluation } \\
\cline { 2 - 5 } & Power(uw) & Delay(ps) & Energy (PDP) & No.T \\
\hline Hamming Encoder with CMOS & 34.4 & 72 & $2477 \mathrm{e}^{-18}$ & 72 \\
\cline { 1 - 1 } Hamming Encoder with GDI & 30 & 80 & $2400 \mathrm{e}^{-18}$ & 40 \\
\cline { 1 - 1 } Hamming Encoder with FS-GDI & 19 & 33.9 & $644.1 \mathrm{e}^{-18}$ & 36 \\
\hline
\end{tabular}

\subsection{The Proposed FS-GDI Hamming Code $(11,7)$ Design Implementing}

The second implemented Hamming code by the FS-GDI for a 7-bit data word, the code word consists of 11-bits i.e. 7-data bits D1, D2, D3, D4, D5, D6, D7 and 4- parity bits P1, P2, P4 \& P8 as shown in Table 2. The parity bits calculated for 7-bit data is as follows in Eqs (4-6):

$$
\begin{aligned}
& \mathrm{P} 1=\mathrm{D} 1 \oplus \mathrm{D} 2 \oplus \mathrm{D} 4 \oplus \mathrm{D} 5 \oplus \mathrm{D} 7 \\
& \mathrm{P} 2=\mathrm{D} 1 \oplus \mathrm{D} 3 \oplus \mathrm{D} 4 \oplus \mathrm{D} 6 \oplus \mathrm{D} 7 \\
& \mathrm{P} 4=\mathrm{D} 2 \oplus \mathrm{D} 3 \oplus \mathrm{D} 4 \\
& \mathrm{P} 8=\mathrm{D} 5 \oplus \mathrm{D} 6 \oplus \mathrm{D} 7
\end{aligned}
$$

The Codeword $: D_{7} D_{6} D_{5} P_{8} D_{4} D_{3} D_{2} P_{4} D_{1} P_{2} P_{1}$. 


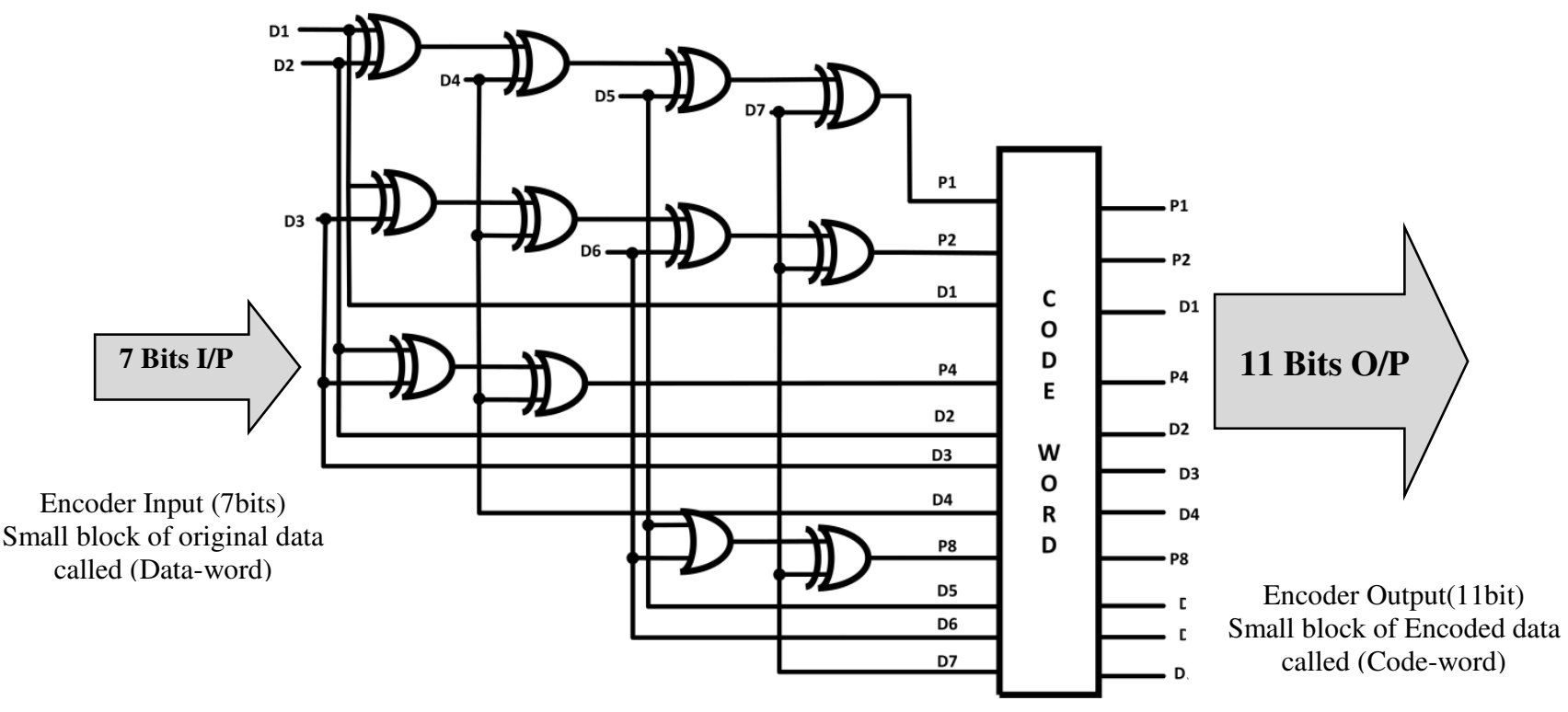

Fig. 4 Hamming Encoder Circuit $(11,7)$ Hardware Contents

\section{a. Performance Evaluating of the Proposed Hamming Encoder (11, 7) Design}

Table 3 Metrics values of the proposed Hamming encoder $(11,7)$ design with respect to the different approaches: Proposed \& Traditional

\begin{tabular}{l|cc|c|c}
\multirow{2}{*}{\multicolumn{1}{c}{ Utilized Approach }} & \multicolumn{4}{|c}{ Metrics of Performance Evaluation } \\
\cline { 2 - 5 } & Power(uw) & Delay(ps) & Energy (PDP) & No.Ts \\
\hline Hamming Encoder with CMOS & 52 & 93 & $5200 \mathrm{e}^{-18}$ & 144 \\
\hline Hamming Encoder with GDI & 45 & 112 & $5715 \mathrm{e}^{-18}$ & 96 \\
\hline Hamming Encoder with FS-GDI & 38 & 64 & $3420 \mathrm{e}^{-18}$ & 72 \\
\hline
\end{tabular}

Table 3 shows the values of the evaluating metrics of the proposed implementing approach of Hamming code $(11,7)$. As shown in the previous experiments which are devoted for evaluating the performance of the proposed design of the different Hamming codes with the various data-word and codeword lengths. The values of the utilized metrics which are measured in these experiments clear the superiority of the Hamming codes implementation based on the FS-GDI compared to the CMOS approach based. 


\subsection{The Proposed FS-GDI Hamming Code (15,11 Design Implementing}

The third implemented Hamming code by the FS-GDI for a 11-bit data word, the code word consists of 15-bits. The input data contains 7-data bits "D1, D2, D3, D4, D5, D6, D7", while the output of the encoder contains 25 bits, it is formed from the 7 data bits and 4- parity bits "P1, P2, P4 \& P8" as shown in Figure ?. The parity bits calculated for 7-bit data is as follows in Eqs (8-11):

$$
\begin{aligned}
& \mathrm{P} 1=\mathrm{D} 1 \oplus \mathrm{D} 2 \oplus \mathrm{D} 4 \oplus \mathrm{D} 5 \oplus \mathrm{D} 7 \oplus \mathrm{D} 9 \oplus \mathrm{D} 11 \\
& \mathrm{P} 2=\mathrm{D} 1 \oplus \mathrm{D} 3 \oplus \mathrm{D} 4 \oplus \mathrm{D} 6 \oplus \mathrm{D} 7 \oplus \mathrm{D} 10 \oplus \mathrm{D} 11 \\
& \mathrm{P} 4=\mathrm{D} 2 \oplus \mathrm{D} 3 \oplus \mathrm{D} 4 \oplus \mathrm{D} 8 \oplus \mathrm{D} 9 \oplus \mathrm{D} 10 \oplus \mathrm{D} 11 \\
& \mathrm{P} 8=\mathrm{D} 5 \oplus \mathrm{D} 6 \oplus \mathrm{D} 7 \oplus \mathrm{D} 8 \oplus \mathrm{D} 9 \oplus \mathrm{D} 10 \oplus \mathrm{D} 11
\end{aligned}
$$

Code Word $: D_{11} D_{10} D_{9} D_{8} D_{7} D_{6} D_{5} P_{8} D_{4} D_{3} P_{8} D_{2} P_{4} D_{1} P_{2} P_{1}$

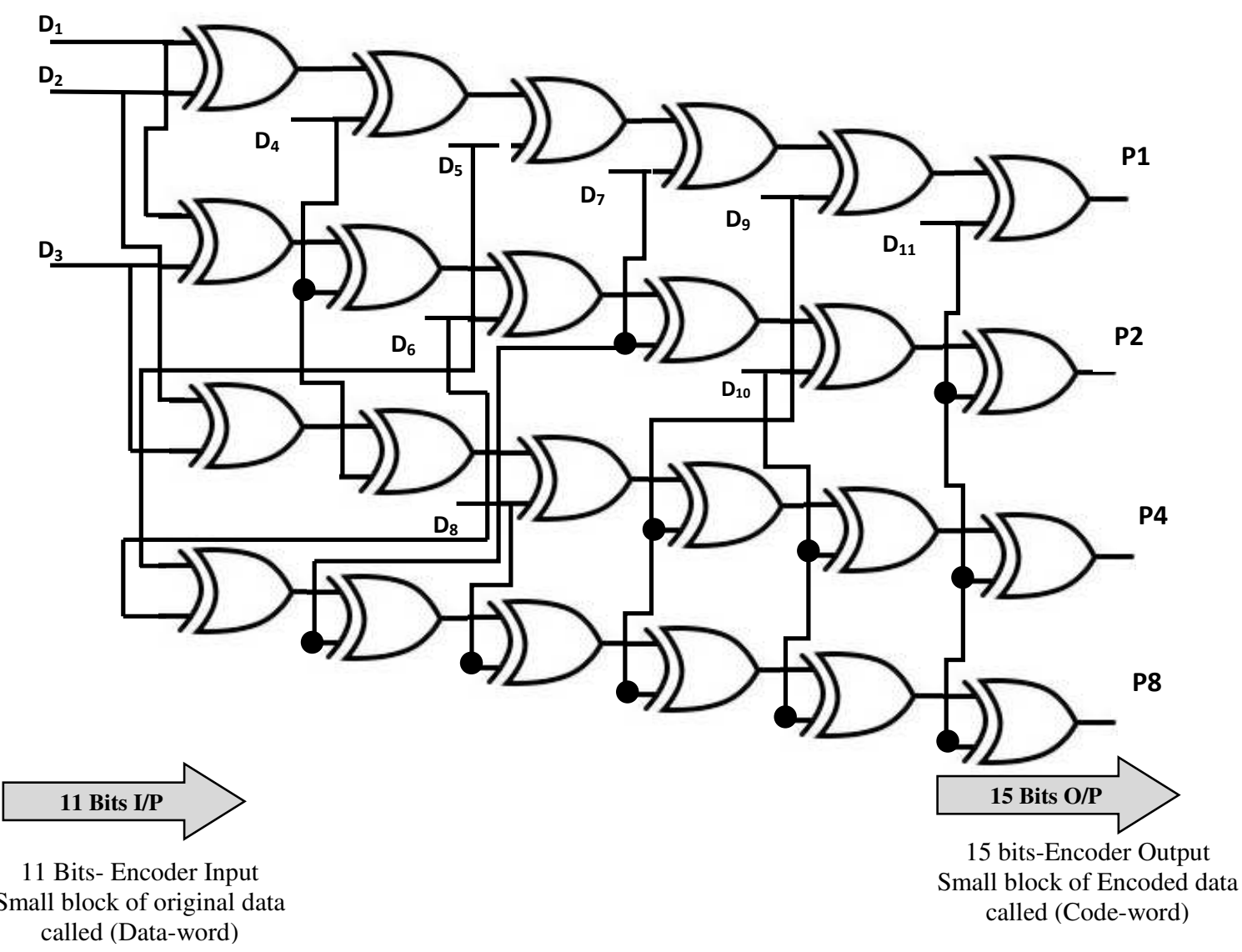

Fig. 2: Hamming Encoder Circuit $(15,11)$ 


\section{a. Performance Evaluating of the Proposed Hamming Encoder $(15,11)$ Design}

In this section the computer-based design is utilized for design and implement the Hamming code $(15,11)$. The Spectre Cadence Virtuoso simulator software package is used for design and executing the simulation experiments the proposed implementing approach of the implemented Hamming codes testing. Table 4 shows the values of the evaluating metrics of the proposed implementing approach of Hamming code $(15,11)$. As shown in the previous experiments which are devoted for evaluating the performance of the proposed design of the different Hamming codes with the various data-word and codeword lengths. The values of the utilized metrics which are measured in these experiments clear the superiority of the Hamming codes implementation based on the FS-GDI compared to the CMOS approach based.

Table 4 Metrics values of the proposed Hamming encoder $(15,11)$ design with respect to the different approaches: proposed \& traditional implementation

\begin{tabular}{l|cc|c|c}
\multirow{2}{*}{\multicolumn{1}{c}{ Utilized Approach }} & \multicolumn{4}{|c}{ Metrics of Performance Evaluation } \\
\cline { 2 - 5 } & Power(uw) & Delay(ps) & Energy (PDP) & No.Ts \\
\hline Hamming Encoder with CMOS & 71 & 100 & $5200 \mathrm{e}^{-18}$ & 288 \\
\hline Hamming Encoder with GDI & 65 & 127 & $5715 \mathrm{e}^{-18}$ & 192 \\
\hline Hamming Encoder with FS-GDI & 49.62 & 73 & $2282 \mathrm{e}^{-18}$ & 144 \\
\hline
\end{tabular}

\section{The Results Analysis}

In this section, the previous experiments results are analyzed numerically. By considering the metrics results of the CMOS approach scenario is the standard and traditional approach, the improving percentage denotes by + , This + symbol means there is amount of improvement. On the other hand the (-) symbol means there is not improving. In Table 4, the results are analyzed numerically as cleared. 
Table 5 Metrics values analyzing of the proposed implementing of Hamming codes based FS-GDI approach compared to the traditional approach

\begin{tabular}{|c|c|c|c|c|}
\hline \multirow{2}{*}{\multicolumn{2}{|c|}{$\begin{array}{c}\text { Hamming Codes \& Implementation } \\
\text { Approaches }\end{array}$}} & \multicolumn{3}{|c|}{$\begin{array}{l}\text { Amount of Improving compared to } \\
\text { Traditional COMS Implementation }\end{array}$} \\
\hline & & \multirow{2}{*}{$\begin{array}{l}\begin{array}{c}\text { Consume } \\
\mathrm{d}(\mathrm{P}) \%\end{array} \\
+12.78 \%\end{array}$} & \multirow{2}{*}{$\begin{array}{c}\text { Delay(ps) } \\
\% \\
-11.11 \%\end{array}$} & \multirow{2}{*}{$\begin{array}{c}\mathrm{H} / \mathrm{W} \\
\text { Simplicity \% } \\
+44.44 \%\end{array}$} \\
\hline \multirow[t]{2}{*}{ Hamming Code $(7,4)$} & $\begin{array}{l}\text { Original } \\
\text { GDI } \\
\text { Approach }\end{array}$ & & & \\
\hline & $\begin{array}{l}\text { FS-GDI } \\
\text { Approach }\end{array}$ & $+44.77 \%$ & $+52.91 \%$ & $+50 \%$ \\
\hline \multirow[t]{2}{*}{ Hamming Code $(11,7)$} & $\begin{array}{l}\text { Original } \\
\text { GDI } \\
\text { Approach }\end{array}$ & $+13.46 \%$ & $-27 \%$ & $+33.33 \%$ \\
\hline & $\begin{array}{l}\text { FS-GDI } \\
\text { Approach }\end{array}$ & $+26.93 \%$ & $+10 \%$ & $+50 \%$ \\
\hline Hamming Code $(15,11)$ & $\begin{array}{l}\text { FS-GDI } \\
\text { Approach }\end{array}$ & $+30.14 \%$ & $+27 \%$ & $+50 \%$ \\
\hline
\end{tabular}

As shown in Table 4, the proposed Hamming codes implementing using the FS-GDI achieves power efficiency $44.77 \%$ and $26.93 \%$ for the Hamming codes $(7,4)$ and $(11,7)$, respectively compared to the CMOS approach. While these proposed codes achieve delay time reduction by $52.91 \%$ and $10 \%$ for Hamming codes $(7,4)$ and $(11,7)$, respectively. These results clear the efficiency of the presented approach for implementing the different error control techniques. In general, the utilizing these proposed implementation approach reduces the power consumption of the data encoding. On the other hand, the Hardware (H/W) of these codes became more simple where the H/W simplicity of the used Hamming codes is reduced $50 \%$ as shown in the results analysis table.

\section{Conclusion}

This paper proposes the FS-GDI approach for implementation the error control schemes for enhancing the power efficiency of the data encoding process. The paper presents proposed implementation of Hamming codes $(7,4),(11,7)$ and $(15,11)$ using FS-GDI approach. The proposed design and simulation experiments are carried out using Cadence Virtuoso simulator package. There are different metrics are utilized for measuring the performance of the proposed FS-GDI based Hamming codes. The simulation experiments 
results prove the superiority of the proposed data encoding tool compared to the traditional approach. The proposed approach for Hamming codes implementation achieves $50 \% \mathrm{H} / \mathrm{W}$ simplicity. Also, the power efficiency of the proposed Hamming codes is improved by $41.77 \%, 23.93 \%$ and $30.14 \%$ for the Hamming codes $(7,4),(11,7)$ and $(15,11)$ respectively compared to the CMOS approach. On the other hand, the delay time is optimized in the proposed Hamming codes implementation approach compared to the traditional CMOS approach. Finally, the proposed Hamming codes in this research paper presents a method for high efficient power data encoding process. This approach can be suitable for implementing the complex error control schemes.

\section{Declaration:-}

- No conflict of interest exists.

Authors wish to confirm that there are no known conflicts of interest associated with this publication and there has been no significant financial support for this work that could have influenced its outcome.

- Also, they confirm that there is no funding was received for this work.

\section{Data Availability:-}

- Author Confirms the data availability which is related to this manuscript in case the requirements for research targets.

- Also, The datasets which is generated during and analyzed during the current research/study are available from the corresponding author on reasonable request.

\section{References}

[1] Mohsen A. M. El- Bendary, "Developing security tools of WSN and WBAN networks applications"; 2015 Springer, Japan.

[2] Yang Guo, Shaolin Xie, Zijun Liu, Lei Yang, Donglin Wang," Parallel Polar Encoding in 5G Communication", 2018 IEEE Symposium on Computers and Communications (ISCC).

[3] Mrinmayi V Patil, Sanjay Pawar, Zia Saquib," Coding Techniques for 5G Networks: A Review", 2020 3rd International Conference on Communication System, Computing and IT Applications (CSCITA).

[4] Yue Wang, Wei Liu, Linquan Fang," Adaptive Modulation and Coding Technology in 5G", 978-1-7281-3129-0/20/\$31.00 @2020 IEEE.

[5] Valerio Bioglio, Carlo Condo, Ingmar Land," Design of Polar Codes in 5G New Radio", DOI 10.1109/COMST.2020.2967127, IEEE Communications Surveys \& Tutorials. 
[6] R. Zimmermann and W. Fichtner, "Low-Power Logic Styles: CMOS Versus Pass-Transistor Logic," vol. 32, no. 7, pp. 1079-1090, 1997.

[7] P. Taylor, W. Al-assadi, A. P. Jayasumana, and Y. K. Malaiya, "Pass-transistor logic design," no. January 2015, pp. 37-41, 2007.

[8] D. Wang, M. Yang, W. Cheng, X. Guan, Z. Zhu, and Y. Yang, "Novel Low Power Full Adder Cells in 180nm CMOS Technology," pp. 430-433, 2009.

[9] P. Kumar and R. K. Sharma, “An Energy Efficient Logic Approach to Implement CMOS Full Adder," J. Circuits, Syst. Comput., vol. 26, no. 05, p. 1750084, 2017.

[10] M. Amini-Valashani, M. Ayat, and S. Mirzakuchaki, "Design and analysis of a novel lowpower and energy-efficient $18 \mathrm{~T}$ hybrid full adder," Microelectronics J., vol. 74, no. November 2017, pp. 49-59, 2018.

[11] V. R. Tirumalasetty and M. R. Machupalli, "Design and analysis of low power high-speed 1bit full adder cells for VLSI applications," Int. J. Electron., vol. 106, no. 4, pp. 521-536, 2019.

[12] MAM El-Bendary, ," FEC merged with double security approach based on encrypted image steganography for different purpose in the presence of noise and different attacks", Multimedia Tools and Applications 76 (24), 26463-26501.

[13] Daniel G. Costa, Solenir Figuerêdo and Gledson Oliveira," Cryptography in Wireless Multimedia Sensor Networks: A Survey and Research Directions”, cryptography journal, MDPI, PP. 1-18, 2017.

[14] JongMin Kim, Hong Sub Lee, Junmin Yi, and Minho Park," Power Adaptive Data Encryption for Energy-Efficient and Secure Communication in Solar-Powered Wireless Sensor Networks", Hindawi Publishing Corporation Journal of Sensors Volume 2016. http://dx.doi.org/10.1155/2016/2678269.

[15] Muhammad Usman, Mian Ahmad Jan, Xiangjian He, Priyadarsi Nanda," Data Sharing in Secure Multimedia Wireless Sensor Networks", IEEE computer society, DOI 10.1109/TrustCom/BigDataS.

[16] Dong Xie, Haipeng Peng, Lixiang Li, Yixian Yang", An Efficient Privacy-preserving Scheme for Secure Network Coding Based on Compressed Sensing", International Journal of Electronics and Communications, 19 May 2017.

[17] Saheed Olanigan, Lei Cao, Ramanarayanan Viswanathan," Rate and power efficient image compressed sensing and transmission", Journal of Electronic Imaging 25(1), 013024 (JanFeb 2016).

[18] Shuo Xiao, Wei Li, Haifeng Jiang, Zhiou Xu, Zihao Hu," Trajectroy prediction for target tracking using acoustic and image hybrid wireless multimedia sensors networks", Multimed Tools Appl. Journal, July, 2017. DOI 10.1007/s11042-017-4846-z 
[19] Nassar SS, Ayad NM, Kelash HM, El-sayed HS, El-Bendary MAM, Abd El-Samie FE, Faragallah OS (2016b) Secure wireless image communication using LSB steganography and chaotic baker ciphering. Wirel Pers Commun 91(3):1023-1049.

[20] Felicia Engmann, Ferdinand ApietuKatsriku, Jamal-DeenAbdulai, Kofi Sarpong Adu-Manu, Frank KatakaBanaseka, "Prolonging the Lifetime of Wireless Sensor Networks: A Review of Current Techniques", Wireless Communications and Mobile Computing Journal, https://doi.org/10.1155/2018/8035065.

[21] S.-H. Shieh, C.-K. Tung, and C.-H. Cheng, "Low-power high-speed full adder for portable electronic applications," Electron. Lett., vol. 49, no. 17, pp. 1063-1064, 2013.

[22] A. Parameswar, H. Hara, and T. Sakurai, "High speed, low power, swing restored passtransistor logic based multiply and accumulate circuit for multimedia applications," pp. 278281, 1994.

[23] Y. Liao, D. Roberts, and E. Hoffman, "VLSI Implementation of a High Performance and Low Power 32-Bit Multiply- Accumulate Unit," Architecture, pp. 3-6.

[24] A. A. Fayed and M. A. Bayoumi, "A merged multiplier-accumulator for high speed signal processing applications," ICASSP, IEEE Int. Conf. Acoust. Speech Signal Process. - Proc., vol. 3, pp. 3212-3215, 2002.

[25] B. Xia, P. Liu, and Q. Yao, "New method for high performance multiply-accumulator design *," vol. 10, no. 7, pp. 1067-1074, 2009.

[26] A. Abdelgawad, "High Speed and Area-Efficient Multiply Accumulate ( MAC ) Unit for Digital Signal Prossing Applications,” pp. 3199-3202, 2007.

[27] S. Shanthala, C. P. Raj, and S. Y. Kulkarni, "Design and VLSI implementation of pipelined multiply accumulate unit,” 2009 2nd Int. Conf. Emerg. Trends Eng. Technol. ICETET 2009, pp. 381-386, 2009.

[28] S. Ahish, Y. B. N. Kumar, D. Sharma, and M. H. Vasantha, "Design of high performance Multiply-Accumulate Computation unit," Souvenir 2015 IEEE Int. Adv. Comput. Conf. IACC 2015, no. 2, pp. 915-918, 2015.

[29] N. Subbulakshmi and R. Manimegalai, "MACGDI: Low Power MAC Based Filter Bank Using GDI Logic for Hearing Aid Applications,” Int. J. Electron. Electr. Eng., vol. 5, no. 3, pp. 235-239, 2017.

[30] N. Kandasamy, F. Ahmad, S. Reddy, R. B. M, N. Telagam, and S. Utlapalli, "Performance evolution of 4-b bit MAC unit using hybrid GDI and transmission gate based adder and multiplier circuits in 180 and $90 \mathrm{~nm}$ technology," Microprocess. Microsyst., vol. 59, pp. 15$28,2018$.

[31] C. F. Law, S. S. Rofail, and K. S. Yeo, "Low-power $16 \times 16-b$ parallel multiplier utilizing pass-transistor logic,” IEEE J. Solid-State Circuits, vol. 34, no. 10, pp. 1395-1399, 1999. 
[32] M. Jhamb, Garima, and H. Lohani, "Design, implementation and performance comparison of multiplier topologies in power-delay space,” Eng. Sci. Technol. an Int. J., vol. 19, no. 1, pp. 355-363, 2016.

[33] R. P., K. P., and A. P., "Modified low power Wallace Tree multiplier using higher order compressors,” Int. J. Electron. Lett., vol. 5, no. 2, pp. 177-188, 2016.

[34] M. Shoba and R. Nakkeeran, "Energy and area efficient hierarchy multiplier architecture based on Vedic mathematics and GDI logic,” Eng. Sci. Technol. an Int. J., vol. 20, no. 1, pp. 321-331, 2017.

[35] S. Mohan and N. Rangaswamy, "An improved implementation of hierarchy array multiplier using CslA adder and full swing GDI logic," Electronics, vol. 21, no. 1, pp. 38-47, 2017.

[36] S. Mohan and N. Rangaswamy, "Area and Energy-Efficient 4-2 Compressor Design for Tree Multiplier Implementation,” Proc. Natl. Acad. Sci. India Sect. A - Phys. Sci., pp. 4-11, 2018.

[37] A. Morgenshtein, A. Fish, and I. A. Wagner, "Gate-diffusion input (GDI): A power-efficient method for digital combinatorial circuits,” IEEE Trans. Very Large Scale Integr. Syst., vol. 10, no. 5, pp. 566-581, 2002.

[38] A. Morgenshtein, I. Shwartz, and A. Fish, "Gate Diffusion Input (GDI) logic in standard CMOS nanoscale process,” 2010 IEEE 26th Conv. Electr. Electron. Eng. Isr. IEEEI 2010, pp. 776-780, 2010.

[39] A. Morgenshtein, V. Yuzhaninov, A. Kovshilovsky, and A. Fish, "Full-swing gate diffusion input logic - Case-study of low-power CLA adder design,” Integr. VLSI J., vol. 47, no. 1, pp. 62-70, 2014.

[40] M. Shoba and R. Nakkeeran, "GDI based full adders for energy efficient arithmetic applications,” Eng. Sci. Technol. an Int. J., vol. 19, no. 1, pp. 485-496, 2016.

[41] G. Ramana Murthy, C. Senthilpari, P. Velrajkumar, and T. S. Lim, "A new 6-Tmultiplexer based full-adder for low power and leakage current optimization,” IEICE Electron. Express, vol. 9, no. 17, pp. 1434-1441, 2012.

[42] T. Bhagyalaxmi, S. Rajendar, and S. Srinivas, "Power-Aware Alternative Adder Cell Structure Using Swing Restored Complementary Pass Transistor Logic at 45nm Technology,” Procedia Mater. Sci., vol. 10, no. Cnt 2014, pp. 789-792, 2015.

[43] MAM El-Bendary, AE Abou El-Azm," Complexity considerations: efficient image transmission over mobile communications channels", Multimedia Tools and Applications 78 (12), 2019. PP. 16633-16664 